Fischer and Orth ${ }^{1}$ quote a value of $563 \mathrm{~m} \mu$ for the $\alpha$-band of a hrmochrome derived from rhodoporphyrin. In view of the values reported here their result appears anomalous and seems to approximate that of the ester rather than of the acid.

Department of Organic Chemistry,

$$
\text { P. S. CLezy }
$$

University of New South Wales.

1 Fischer, H., and Orth, H., Die Chemie des Pyrroles, \&, Part 1, 533 (Akademische Verlagsges., Leipzig, 1937)

"Fischer, H., and Beer, L., Z. physiol. Chemie, 244, 81 (1936).

${ }^{3}$ Conant, J. B., Hyde, J. F., Moyer, W. W., and Dietz, E. M., J. Amer. Chem. Soc., 53, 359 (1931)

- Morell, D. B., Barrett, J., and Clezy, P. S., Biochem. J., 78, 793(1961).

'Barrett, J., and Clezy, P. S., Nature, 184, 1988 (1959).

\section{Examination of Side-Chain Interactions in Poly-L-Tryptophan}

WHILE hypochromism has been examined quentitatively by Tinoco ${ }^{1}$ for polynucleotides and Imahori and Tanaka ${ }^{2}$ have reported decreased extinetion of the peptide absorption band at $1900 \AA$. in poly-Lglutamic acid in the helical conformation, it was of interest to examine interactions of the aromatic sidechains of poly-L-tryptophan. The results of absorption and fluorescence studies of the polyamino-acid comparod with a suitable model for the monomer are discussed in this communication.

Poly-L-tryptophan, having an average molecular weight of 17,800, was supplied by Dr. Michael Sela. Acetyltryptophan is commercially available and tryptophan ethyl ester was prepared by a modification of the method of Brenner and Hubor ${ }^{8}$. Solvents were distilled prior to use except for dimethylformamide, which was 'Spectroquality' reagent purchased from Matheson, Coleman and Bell. Absorption spectra were taken with a Cary recording spectrophotometer model 14, and intensities of emitted light were measured with the Aminco-Bowman fluorometer with the monochrometers set at constant excitation and emission wave-lengths.

The limited solubility of polytryptophan did not permit examination over a wide range of solvent polarities. In this work the solvents used were dimethylformamide, dimethyl sulphoxide and $n$ butylamine/water ( $9: 1$ by vol.). Poly-L-tryptophan absorption obeys Beer's law in these solvents over the twenty-fold range of concentration studied. The absorption maxima and their extinction coefficients are shown in Table 1 togother with the results of Hurd and Bauer ${ }^{4}$ for polytryptophan (average molecular weight 4,000) in ethanol.

The total areas under the absorption curves as a measure of the oscillator strength, $f$, were determined after extrapolation below $2600 \AA$., where accurate measurements were not obtainable because of solvent absorption. The $f$ 's are very nearly the same for

Table 1. Absorption Maxima and Extinction Coefficients of Table 1. ABSORPTION MAXIMA AND EXTTRCTION Co
N-ACETYL- AND POLY-TRYPTOPHAN

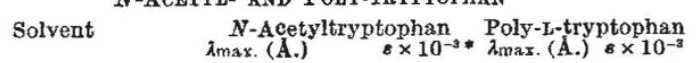

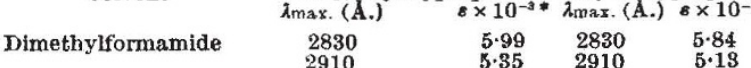

Dimethyl sulphoxide $\quad 2835 \quad 5 \cdot 68 \quad 2835 \quad 5 \cdot 74$

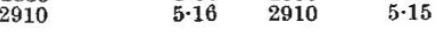

$\begin{array}{ccccc}\text { n-Butylamine/water } & 2840 & \mathbf{5 . 5 8} & 2840 & \mathbf{5 . 4 3} \\ \text { (9:1 by volume) } & 2915 & \mathbf{5 . 0 7} & 2915 & \mathbf{5} \cdot 10\end{array}$

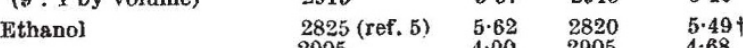

* These values are the average of 10-15 determinations and are

\pm 0.05
+ Values from Hurd and Bauer (ref. 4) for polytryptophan, the average molecular weight of which is 4,000 . acetyl- and poly-tryptophan in the solvents used in this investigation. Similarly, temperature variation between $25^{\circ} \mathrm{C}$. and $80^{\circ} \mathrm{C}$. had no exclusive effect on the absorption spectrum of the monomer or polymer.

Although there are no significant differences between the absorption properties of acetyl- and poly-tryptophan, tryptophan-tryptophan interactions may be detected more readily by the fluorescence properties of the aromatic amino-acid side-chain. Emission intensities in the $3400 \AA$. region were measured for solutions having optical densities of $0.050-0.250$ at the absorption maximum. In all solvents, the polymer was consistently 35-45 per cent less fluorescent than either acetyltryptophan or tryptophan ethyl ester in the same solvent. The decreased fluorescence of the polymer may be a reflexion of quenching as a consequence of the high local concentration of the aromatic groups.

The absence of hypo- or hyper-chromic effects in the absorption of polytryptophan suggests that either the transition moments of the freely rotating aromatic side-chains are not oriented or interactions between identical transitions cannot be detected by absorption studies. The present work also indicates that the high quantum yield of some tryptophancontaining proteins is not explicable in terms of tryptophan stacking as has been suggested ${ }^{6}$. Borrowing from or lending energy to other groups in the protein may account for unusual fluorescent properties of some albumins as recently reported by Weber?

National Cancer Institute, SIDNEY SHIFRIN

National Institutes of Health, Bethesda.

'Tinoco, jun., I., J. Amer. Chem. Soc., 82, 4785 (1960).

2 Imahori, K., and Tanaka, J., J. Mol. Biol., 1, 359 (1959).

'Brenner, M., and Huber, W., Helv. Chim. Acta, 36, 1109 (1953).

- Hurd, C. D., and Bauer, L., J. Org. Chem., 18, 1440 (1953).

${ }^{5}$ Edwards, B. G., Arch. Biochem., 21, 103 (1949).

- Velick, S. F., J. Biol. Chem., 233, 1455 (1958).

'Weber, G., Biochem. J., 79, 29 P (1961).

\section{Absence of Correlation between Odour and Molecular Vibration}

IN 1928 Dyson $^{1}$ proposed a relationship between odour and molecular vibration. This hypothesis was worked out in more detail by Wright ${ }^{2-6}$, who correlated the odour of a compound with its low-frequency vibrations (below $800 \mathrm{~cm}^{-1}$ ).

Wright postulates that odour-perception is caused by the interaction between the odorous molecule and a pigment molecule present in the olfactory cell. The former is attrched to the latter by "forces of adsorption, or something of the sort" 5 . The pigment molecule is permanently in an excited electronic state and can only fall back to its electronic ground-state if the relevant selection rule is relaxed by a perturbation due to the odorous molecule in "a process akin to Fermi-resonance". This perturbation is thus assumed to occur only if the odorous molecule and the receptor molecule have one or more identical vibration frequencies. The electronic transition then triggers an electrical process in the cell membrane. Leaving the electronic transition and its selection rules out of discussion in this communication, we will now examine the vibrational interactions more closely.

It is easily seen that no correlation can be found between the vibrations of a free molecule and its odour if the molecule forms a complex with the receptor molecule, as the vibrational frequencies of 